Published in final edited form as:

Cancer Res. 2013 November 15; 73(22): 6839. doi:10.1158/0008-5472.CAN-13-2936.

\title{
Retraction: Modulation of CD59 Expression by Restrictive Silencer Factor-Derived Peptides in Cancer Immunotherapy for Neuroblastoma
}

The article titled "Modulation of CD59 Expression by Restrictive Silencer Factor- Derived Peptides in Cancer Immunotherapy for Neuroblastoma," which was published in the July 15, 2008, issue of Cancer Research (1), is being retracted at the request of the authors following the release of the conclusions of an internal investigation panel established by Cardiff University to examine allegations of research misconduct in the preparation of the manuscript. The panel found evidence of splicing or pasting affecting Fig. 2A, without indication that this had been done. The panel feels that although this image manipulation cannot be characterized as "fabrication" because there is no reason to doubt the validity of the underlying science in the article, it represents unacceptable practice when submitting a manuscript for publication. Dr. R.M. Donev accepted full responsibility for the action. The panel concluded that none of the other coauthors of this manuscript knew, or had reason to suspect, that the data presented in the manuscript had been manipulated by Dr. R.M. Donev.

Five of the six authors agreed to this Retraction. Attempts to contact the first author, Rossen M. Donev, were unsuccessful.

Baalasubramanian Sivasankar, Timothy R. Hughes, B. Paul Morgan

Department of Medical Biochemistry and Immunology, School of Medicine, Cardiff University, Cardiff, United Kingdom

Lisa C. Gray, Carmen W. van den Berg

Department of Pharmacology, Therapeutics and Toxicology, School of Medicine, Cardiff University, Cardiff, United Kingdom

\section{References}

1. Donev RM, Gray LC, Sivasankar B, Hughes TR, van den Burg CW, Morgan BP. Modulation of CD59 expression by restrictive silencer factor-derived peptides in cancer immunotherapy for neuroblastoma. Cancer Res. 2008; 68:5979-87. [PubMed: 18632654] 